\title{
Postulate einer Eiszeit-Theorie
}

\author{
Von D. D. Kvasov, Leningrad
}

$\mathrm{Z} u$ s a m me n f a s u n g. Einige Grundvorstellungen der Quartärgeologie können als Postulate der Vereisungstheorie genannt werden. Es wird versucht, über ein Postulatensystem die Ursachen der Vereisung zu erklären. Dabei ergeben sich drei Fragen:

1. Warum war es im Pliozän und im Quartär bedeutend kälter als vorher? Wahrscheinlich war in dieser Zeit die Wasserzirkulation zwischen dem Atlantischen Ozean und dem Nördlichen Eismeer sehr vermindert, was zur Abkühlung des letzteren führte. Der Aufbau des Island-FaroerBasaltmassivs führte zur Abschwächung der Zirkulation. Die Abkühlung mußte zwangsläufig zur Vereisung führen. Aber es bleibt unklar, warum die Vereisung der Mittelbreiten erst vor einigen hunderttausend Jahren begann und sogleich große Ausmaße erreichte. In Zusammenhang damit steht die zweite Frage:

2. Was verhinderte die Vereisung der Mittelbreiten während des Zeitabschnitts vor ca. 0,5-3,5 Millionen Jahren? Man kann vermuten, daß in diesem Zeitabschnitt die Regression des Ozeans stattfand und sehr bedeutende Schelfflächen sich in Land umwandelten. Das Land am Platz des Barentmeeres war wahrscheinlich von einem Eisschild eingenommen. Der über ihm herrschende Antizyklon führte zur Vergrößerung der Kontinentalität des Klimas in Nordeuropa. Dort entstanden Verhältnisse, die für den Aufbau eines Eisschildes ungünstig waren. Analoge Verhältnisse herrschen heute im Nordteil von Kanada. Dort verhindert der Antizyklon über dem Grönländischen Eisschild den Eisaufbau.

3. Wie kann man die nachfolgenden glazialen und interglazialen Epochen erklären? Bei dem Sinken der Temperatur auf $1-2^{\cup}$ in Skandinavien entsteht ein kleiner Eisschild, über dem sich eine kalte Luftmasse bildet, welche die Umgebung abkühlt. Unter der Wirkung dieser Abkühlung vergrößert sich der Schild. Die Abkühlung erfaßt große Flächen der Erde und löst das Wachstum der Gletscher in Nordamerika und anderen Gebieten aus. Die Bindung großer Wassermengen in den Gletschern führt zur Absenkung des Weltmeeres. Die Schelfflächen werden wieder trocken und es entsteht die Möglichkeit der Wiederherstellung des Barent-Eisschilds. Unter seiner Wirkung nimmt die Zufuhr der Niederschläge auf die östlichen und südöstlichen Bereiche des skandinavischen Eisschildes sehr ab, welcher sich rasch verkleinert. Dieses führt zur Verkleinerung der anderen Eisschilde der Mittelbreiten. Der Meeresspiegel steigt an; es wächst die Intensität der Eisbergbildung an der Grenze des Barent-Eisschilds, der sich auch verkleinert. Es beginnt ein Interglazial, und dadurch entstehen die Bedingungen zur Bildung eines neuen Glazials. Die Aufeinanderfolge von Glazialen und Interglazialen ist ein autozyklischer Prozeß. Die Abkühlungen, die durch periodische Veränderung der Erdbahnelemente bedingt sind (Milankovitch-Kurve), fördern diesen Prozeß.

Die Glaziale werden periodisch wiederkehren. Man kann hoffen, daß aufgrund der heute entwickelten Theorie der allgemeinen Zirkulation der Atmosphäre und des Ozeans die Klimate der geologischen Vergangenheit rekonstruiert und auch die Wege der Auswirkung auf das Klima angedeutet werden können. Die vorliegende Theorie mißt den Schwankungen des Niveaus, der Zirkulation, den Temperatur- und Eisverhältnissen des Weltozeans eine große Bedeutung bei. Darum kann sie die ozeanologische Theorie der Vereisung genannt werden.

Abstract. Certain fundamental propositions of Quaternary geology may be termed postulates of a theory of Ice Ages. The article is an attempt to show that a system of postulates makes it possible to explain the causes of glaciation. The question of the causes of glaciation may be subdivided into three:

1) Why were the climates in the Pliocene and Quaternary colder than in the preceding geologic periods? The water circulation between the Atlantic and the Arctic oceans at that time must have been weaker, which resulted in the cooling of the Arctic. The reduction of circulation was promoted by the growth of the Icelandic-Faroer basalt massif. It was inevitable that the cooling would have led to glaciation. It is not clear, however, why the glaciation of temperate latitudes began only a few hundred thousand years ago and immediately attained vast proportions. This gives rise to a second question:

2) What prevented glaciation in temperate latitudes in the interval of $\sim 3,5-0,5 \mathrm{mln}$ years ago? It can be supposed that there was a regression of the oceans at that time and vast expanses of the shelves became dry land. The land on the site of the Barents Sea may have been occupied by an ice sheet. The anticyclone prevailing over it enhanced the continental climate of nothern 
Europe, making the conditions there unfavourable to glaciation. Similar conditions are now to be found in northern Canada where glaciation is prevented by the anticyclone over the Greenland ice sheet.

3) What accounts for the alternation of glacial and interglacial stages? With a slight lowering of temperature (by $1-2^{\circ}$ ) in Scandinavia a small ice sheet is formed there and the air mass established over it cools the territories around the sheet. The cooling effect of this air mass causes the ice sheet to grow in size. The cooling extends to large areas of the earth and brings about the growth of glaciers in North America and in other regions. The accumulation of enormous water masses in glaciers results in the lowering of sea level. The shelves become dry land again and conditions are created for the recovery of the Barents ice sheet. Under its influence nourishment is reduced for the eastern and south-eastern peripheries of the Scandinavian ice sheet which begins quickly to retreat. This brings about the retreat of other ice sheets in temperate latitudes. The sea level rises; the formation of icebergs is enhanced in the periphery of the Barents ice sheet which also begins to retreat. Interglacials begin, which creates conditions for a new glacial. The alternation of glacials and interglacials is an autofluctuational process. Its emergence is promoted by the cooling caused by periodic changes of elements of the Earth's orbit (the Milankovitch curve).

Ice Ages will be periodically repeated in future. It is to be hoped that the theory of the general circulation of the atmosphere and the oceans being developed at present will make it possible to calculate the climates of the geologic past and to outline the ways of influencing the climate.

The theory proposed stresses the importance of the fluctuations in sea level, circulation, thermal and ice regime of the oceans. Therefore, it can be called an oceanological theory of Ice Ages.

\section{Einleitung}

Das Quartär ist auf Grund der Verteilung von Land und Meer für die Ausbildung von Eiszeiten prädestiniert. Das Südpolargebiet wird vom Antarktischen Kontinent eingenommen, der mit einer riesigen Eismasse bedeckt ist. Der Arktische Ozean ist größtenteils von Land umgeben, so daß seine Wassermassen nur in geringem Maße an der Zirkulation des Weltmeeres beteiligt sind. Diese Verteilung ermöglicht die scharfe Klimadifferenzierung der äquatorialen und polaren Breiten. Sie begünstigt die Vereisung in den polaren Breiten und in dem Nordteil der gemäßigten Klimazone.

Seit Beginn des Palaeogens erfolgt eine generelle Abkühlung. Sie fällt zusammen mit dem Aufbau des Island-Faroer-Basaltmassivs, der spätestens im Eozän begann und der bis in die Gegenwart anhält. Island und die Faroer sowie das riesige submarine Plateau, das Island und die Insel-Gruppe umgibt, hindern den Golfstrom daran, ins Eismer weiterzufließen. Man könnte daraus schließen, daß vor einigen Millionen Jahren in den polaren Breiten eine Vereisung ausgelöst wurde, die sich in der nachfolgenden Zeit auch auf die gemäßigte Klimazone ausgedehnt hätte. Die Vereisung setzte dort aber erst vor wenigen hunderttausend Jahren ein, mit einem nachfolgenden Wechsel von Eiszeiten und Interglazialen. Die Erklärung muß deshalb komplizierter sein.

Es ist zu hoffen, daß in naher Zukunft die Dynamik der Atmo- und Hydrosphaere sowie der Gletscher berechnet werden kann, daß daraus Interpretationen der naturgeschichtlichen Veränderungen zumindest für den Zeitabschnitt der letzten Millionen Jahre möglich sind. Einige Grundvorstellungen und -erkenntnisse der Quartärgeologie müssen für solche Kalkulationen die Basis bilden. Diese Grundvorstellungen kann man als Postulate einer Eiszeitheorie bezeichnen.

\section{Die Maclaren- und A. Penck/Hollin-Postulate}

Untersucht man die Ursachen der Vereisung, so müssen die Beziehungen zwischen Vereisung und dem Meeresspiegel berücksichtigt werden. Der Meeresspiegel erniedrigt sich bei einer Bindung des Wassers in größeren Eismassen (Maclaren 1841). Diese Erkenntnis, die als Maclaren-Postulat bezeichnet wird, ist allgemein bekannt. 
Viel weniger bekannt ist die Erkenntnis von der Abhängigkeit der Vereisungen vom Niveau des Meeresspiegels. Abgesehen von der Ablation ist die Eisbergbildung ein sehr wichtiger Faktor der Massenbilanz des Eises in den Polargebieten. Seine Wirksamkeit wird von der Lage des Meeresspiegels beeinflußt. Bei einer Absenkung vermindert sich die Eisbergbildung, und die Eismasse dehnt sich bei positiver Massenbilanz bis zur entsprechenden Küstenlinie aus. Die Ausdehnung der Vereisung in den Polargebieten hängt deshalb nicht von den Klimaänderungen $a b$, die eine Vereisung über einen langen geologischen Zeitabschnitt hinweg begünstigen, sondern von den Meeresspiegelschwankungen. Diese Annahme wurde von PeNCK (1928) für die Antarktis begründet. Später wurde sie von Hollin (1962) weiterentwickelt. Sie wird deshalb als A. Penck/Hollin-Postulat bezeichnet.

\section{Das Dana/Nansen-Postulat}

DANA (1855) nahm eine beträchtliche Landhebung im Praeglazial an, welche die Vereisung ausgelöst hätte. NANSEN (1904) bestätigte die Annahme einer großen Regression des Weltmeeres an der Grenze Neogen/Quartär. Das Dana/Nansen-Postulat ist bis in die Gegenwart viel diskutiert worden. So wurde versucht, die anomal tiefen, schelfähnlichen, submarinenFlächen als Kontinent-Flexuren zu deuten (Holtedahl 1958). Marine Terrassenflächen an vielen Küsten führten zur Annahme, daß der Meeresspiegel im Neogen höher gelegen habe als heute (TANNER 1968). Diese Terrassen liegen jedoch allesamt in tektonisch gehobenen Gebieten (GrEGORY 1931).

Die jüngsten Daten über das Untergrundrelief der quartären Ablagerungen bekräftigen das Dana/Nansen-Postulat. Auf den ausgedehnten Flächen, welche die Nordküste Eurasiens - von Großbritannien bis Chukotka - umsäumen, wurden durch Bohrungen und geophysikalische Untersuchungen enge Täler nachgewiesen, die mit quartären Sedimenten gefüllt sind und deren Talsohlen mehrere hundert Meter tief unter das Niveau des heutigen Meeresspiegels hinabreichen (Blundell u. a. 1969; CePEK 1967; RüHLE 1965; Malachovski \& Markov 1969; Nikonov 1967; Troizky 1966; Baranova u. a. 1968). Der Erosionszyklus, der durch die Absenkung des Meeresspiegels ausgelöst wurde, führte auch zur Ausbildung einiger begrabener Täler im Zentrum der Osteuropäischen Ebene (SHIK 1961) und im Südteil von Westsibirien (Volkov u. a. 1969).

Auf Grund der Ergebnisse aus dem Nordseebecken kann der Erosionszyklus datiert werden. Danach fällt er in die Zeit zwischen die Ablagerung der Coralline- und Red CragSedimente bzw. Scaldisian- und Poederlian-Schichten (niederländische Stratigraphie), d. h., genau an die Grenze zwischen Neogen und Quartär (VAlentin 1951/52; van VoorthuYSEN 1954).

In einigen Gebieten kann die Zertalung durch tektonische Bewegungen ausgelöst worden sein. Ihre weite Verbreitung in Gebieten mit unterschiedlicher tektonischer Struktur spricht jedoch mehr für eine Auslösung durch Absenkung des Meeresspiegels. In der Regel sind diese Täler sehr eng und von V-förmigem Querschnitt. Diese Formen weisen auf eine kurze Zeitdauer der Regression hin.

Die Annahme einer relativen Landhebung in Beziehung zum Meeresspiegel wird auch von Markov \& Lazukov (1965) und von anderen Autoren bekräftigt, nicht aber von GoRETSKY (1967). Wir kennen Spuren der Transgression auf dem Meeresboden. Der gesamte Schelf vor der Nordküste von Eurasien (GAKKEL \& DibNer 1967) sowie vor Island und Labrador gehörte einst zum Festland. Im Ochotskischen Meer wurden in einer Tiefe von mehreren hundert Metern unter dem Meeresspiegel alte Strandlinien gefunden (Udinzev 1957). Anzeichen für einen niedrigen Stand des Meeresspiegels im ausgehenden Neogen sind auch von den Küsten Australiens und Neuseelands bekannt (GLENIE u. a. 1968). Es ist die Möglichkeit nicht ausgeschlossen, daß einige sogenannte Kontinental- 
Plateaus während der Regressionsperiode durch Abrasion entstanden sind. Bohrungen auf dem Plateau Blake bei Florida wiesen Oberflächen aus Oligozän und teilweise aus Miozän nach, während Pliozän-Ablagerungen vollständig fehlen (EMERY \& ZARUDSKi 1967).

Die spätneogene Regression der Ozeane kann nur durch eine Absenkung großer Gebiete des Meeresbodens ausgelöst worden sein. Von dieser Absenkung zeugen die Guyots. Ihre Gipfel liegen etwa $1500 \mathrm{~m}$ unter dem Meeresspiegel. Bislang gibt es nur wenige Indikationen für das Alter der Abrasionsflächen auf den Guyots. Gedregte Gesteinsproben ergaben ein Alter von Oberkreide bis Neogen (Menard 1964). Die Denudationsfläche auf diesen Gesteinen ist jedoch jünger. Guyots bildeten sich zu verschiedenen Zeiten; im Mittleren Papifik können sie relativ alt sein (Hamilton 1956). Die jüngsten Absenkungen erfolgten im Östlichen Pazifik sowie in anderen Ozeangebieten. Der nachfolgende Anstieg des Meeresspiegels könnte mit dem Aufbau des Mittelozeanischen Rückens zusammenhängen.

Möglicherweise steht die Regression in Beziehung zu den beiden letzten Zyklen der Ausweitung des Meeresbodens (J. Ewing \& M. Ewing 1967). Die Anlage des Mittelozeanischen Rückens (erster Zyklus) kann mit der Regression verbunden werden und der Aufbau (zweiter Zyklus) mit der Transgression. Der letzte Zyklus fällt in die Zeit vor ca. 10 Mio. Jahren. Zu dieser Zeit entstanden auch die Rift-Systeme vom Baikal-Typ (ArTemyev \& Artyushoov 1968). Nach stratigraphischen Untersuchungen fanden die intensivsten tektonischen Bewegungen im Baikalgebiet (Uflyand u. a. 1969), im Gebiet des Toten Meeres (NeEv \& EMEry 1967) und in Ostafrika (Girdler u. a. 1969) am Ausgang des Neogens statt. Daraus ergibt sich für den Beginn des letzten Zyklus der Meeresboden-Ausweitung ein jüngeres Alter kurz vor Beginn des Quartärs, d. h. ein Alter von etwa 3,5 Mio. Jahren (BANDY 1967, Gromov u. a. 1969).

\section{Paläogeographie des Eopleistozäns}

\section{Das Gromov-Postulat}

Nach dem A. Penck/Hollin-Postulat begünstigt eine Absenkung des Meeresspiegels sehr die Vereisung in den Polargebieten. Der Antarktische Eisschild, der im Neogen aufgebaut wurde, erreichte seinen größten Umfang während der Zeit der größten Regression. Nach paläomagnetischen Messungen erreichte die Vereisung in der Antarktis ihr Maximum vor 3,35-2,35 Mio. Jahren (Goodel u. a. 1968). Die Absenkung des Meeresspiegels und der Einfluß der Vereisung in der Antarktis lösten die Vereisung in Patagonien aus, deren absolutes Alter mit ca. 3 Mio. Jahren angegeben wird (Mercer 1969). In Californien gibt es Indikationen für eine gleichaltrige Gebirgsvergletscherung (Curry 1966).

Im Nördlichen Eismeer hatten sich Eisberg-Sedimente schon vor 4 bis 6 Mio. Jahren abgelagert, was die Vereisung des sich vergrößernden Festlandes zeigt (STEUERwald u. a. 1968; Herman 1970). Die Regression des Weltmeeres bewirkte in den Küstengebieten des Nordatlantiks und des Eismeeres einen großen Festlandszuwachs. Das Norwegisch-Grönländische Becken und das Nordpolarbecken waren größtenteils isoliert. Sie waren nur durch die engen Straßen zwischen den Faroes und den Shetland-Inseln sowie zwischen Grönland und Spitzbergen mit dem Atlantik verbunden. Das warme Wasser des Atlantiks konnte diese Straße kaum passieren. Infolgedessen kam es zum Aufbau des Grönländischen Eisschildes, dessen Umfang auch heute noch von der Lage des Meeresspiegels bestimmt wird. Der gegenwärtige Zuwachs an Niederschlägen auf der Eisoberfläche beträgt nach BAUER (1955) $446 \mathrm{~km}^{3}$ pro Jahr, bei einem Massenverlust von $530 \mathrm{~km}^{3}$, wovon $315 \mathrm{~km}^{3}$ auf die Ablation und $215 \mathrm{~km}^{3}$ auf die Eisbergbildung entfallen. Bei einem schnellen Absinken des Meeresspiegels würde die Eisbergbildung gestoppt. Die nunmehr positive Massenbilanz 
würde zu einem Eiszuwachs führen, solange bis der Eisschild die neue Küstenlinie erreicht hat. Auf Island gibt es heute einige kleine Eisschilde. Zu Beginn des Quartärs war die Insel teilweise vergletschert (WENSINK 1965). Andere Gebiete mit rezenter Vergletscherung sind die Archipele im Nördlichen Barentsmeer, namentlich Spitzbergen, Franz-Joseph-Land und Novaja Semlja. Diese kleinen Eisschilde wachsen nur deshalb nicht weiter, weil ihre Verbreitung durch die Eisbergbildung und das Abtauen im Kontaktbere:ch mit dem Meerwasser begrenzt wird. Dieser Massenverlust beträgt auf Franz-Joseph-Land $40 \%$ (KRENKE 1964) und auf Novaja Semlja 15\% der Gesamtbilanz (Chizhov u. a. 1968). SchneeAkkumulation und -Ablation halten sich in diesen Gebieten etwa die Waage; die Gletscher verkleinern sich infolge der Eisbergbildung. Wären diese Eisschilde nicht vom Meere begrenzt, dann würden sie in den kurzen wärmeren Klimaabschnitten, wie dem gegenwärtigen, ihren Umfang beibehalten. In kälteren Klimaabschnitten würden sie einen großen Eisschild bilden, der große Teile des Barents- und Karameeres einnehmen würde. Dies muß in den Intervallen vor ca. 3,5-0,5 Mio. Jahren der Fall gewesen sein.

Der Aufbau der Eismassen in der Antarktis, in Grönland, im Barentsmeer und in anderen Gebieten bedingte eine weitere Absenkung des Meeresspiegels und der Wassertemperatur im Meer. Das hatte eine generelle Klimaverschlechterung zur Folge. Im Eopleistozän (Terminologie nach Gromov entspricht dem Altpleistozän in Westeuropa und Nordamerika) reichte das für eine Vereisung in der gemäßigten Klimazone noch nicht aus. Stationäre Antizyklone über den Eisschilden von Grönland und des Barentsmeeres, die große Eisdecke der Norwegischen See, trockengefallenes Land im Bereich der Ostsee und in großen Gebieten der Nordsee verursachten in Europa und Nordamerika ein kontinentaleres Klima und verhinderten so die Vereisung. Diese Verhältnisse sind vergleichbar mit denen der kältesten Phasen des Pleistozäns in Ostsibirien, wo es infolge des kontinentalen Klimas nur zu geringen Vereisungen kam.

Die klimatischen Verhältnisse im Eopleistozän können auf Grund von paläontologischen (Gromov u. a. 1969) und paläobotanischen Ergebnissen (GRICHUK 1959) beurteilt werden. Zur Rekonstruktion der paläogeographischen Verhältnisse dieser Zeit trug besonders Gromov (1948) bei. Er bestätigt die Annahme, daß es im Eopleistozän in der gemäßigten Klimazone keine Vereisung gab und daß das Klima zu dieser Zeit relativ kalt und kontinental war (im Vergleich zum Klima des vorigen Zeitalters). Diese Annahme wird als Gromov-Postulat bezeichnet.

Während des Eopleistozäns erreichte der Kaspisee mit der Aktschagyl-Apscheron-Transgression seinen höchsten Stand, nachdem er vorher seinen tiefsten Stand hatte. Der See, der auch während der Transgression ohne Abfluß blieb, enthielt eine sehr spezifische, endemische Fauna. Bislang sind die Ursachen der Transgression noch ungeklärt. Tektonische Bewegungen können wegen der Abflußlosigkeit des Beckens nicht dafür in Frage kommen (Kvasov 1966). Das Klima war damals nicht humider als vorher. Es verbleibt zur Erklärung lediglich die Annahme einer beträchtlichen Vergrößerung des Einzugsgebietes. Solange der Schelf im Barentsmeer zum Festland gehörte, müssen die Mündungen der von der Osteuropäischen Ebene nach Norden gerichteten Flüsse in der Nähe von Spitzbergen gelegen haben. Der Barents-Eisschild blockierte die Flüsse und zwang sie, nach Süden abzufließen (Kvasov u. a. 1969). Wahrscheinlich vereinigten sie sich im „YergenFluß", der die oberen Portionen der "Yergen-Sande“ aufschüttete und in den Kaspisee mündete (VASILYEV 1969).

Aus dem Bereich des Schwarzen Meeres ist eine größere Regression nicht bekannt. Im Eopleistozän war dort ein Seebecken. Anzeichen für eine tiefreichende Regression finden sich im Mittelmeergebiet (Chumakov 1967). Sie wird dem Plesancien zugeordnet. Es ist noch unklar, weshalb diese Transgression früher erfolgte als die in der Nordsee. Das kann aber mit spezifischen Besonderheiten im Mittelmeerraum zusammenhängen. Vielleicht war 
die Verbindung des Mittelmeeres mit dem Atlantik über die Straße von Gibraltar zeitweilig unterbrochen, analog den Verhältnissen im Roten Meer während des Jungpleistozäns (Kvasov 1969). Wahrscheinlich muß auch die stratigraphische Korrelation zwischen den entsprechenden Ablagerungen des Mittelmeer- und Nordseegebietes revidiert werden.

\section{Paläogeographie des Pleistozäns}

\section{Die Brooks- und Nehring/Grichuk-Postulate}

Im Pleistozän ('Terminologie nach Gromov entspricht dem Mittel- und Jungpleistozän in Westeuropa und Nordamerika) kam es zu einem Anstieg des Meeresspiegels, was den Barents-Eisschild verschwinden ließ (nach A. Penck/Hollin-Postulat). An der Nordküste von Eurasien erreichte der Meeresspiegel seinen heutigen Stand. In einigen Gebieten überschritt er ihn sogar (Petrov 1969). Die Eisschilde in der Antarktis und in Grönland blieben dabei aber erhalten. Sie beeinflußten das Klima weiterhin in beträchtlichem Maße. Aus den theoretischen Überlegungen von Вrоокs (1950) ergibt sich, daß eine geringe Temperaturniedrigung genügt, um einen Eisschild aufzubauen, der so groß ist, daß die Luftemperaturen darüber relativ niedrig bleiben. Somit kann sich der Eisschild rasch vergrößern, bis seine südlichen Ausläufer Gebiete mit hoher Ablation erreichen, in denen der Vorstoß dann zum Stillstand kommt.

Der skandinavische Eisschild verstärkte die Abkühlung, die zu seinem Aufbau führte, beträchtlich, so daß es zu einer Klimaverschlechterung in der gesamten gemäßigten Klimazone der Nordhalbkugel und zum Aufbau von Eisschilden in Nordamerika, NW-Sibirien und anderen Gebieten kam. Die Vereisungen in der gemäßigten Klimazone können folglich a.uf der Grundlage von Annahmen erklärt werden, die man als Brooks-Postulat bezeichnen kann.

Eine Temperaturerhöhung während des Maximums einer Vereisung wird wegen der Schutzwirkung der hohen Albedo nicht sofort eine Verkleinerung des Vereisungsgebietes zur Folge haben. Deshalb haben einige Wissenschaftler eine Theorie der autozyklischen Prozesse entwickelt, nach der die Gletscher selbst Anlaß zu ihrem Abschmelzen geben. Nach der Theorie von M. EwIng \& Donn $(1956,1958,1966)$ beginnt die Vereisung, wenn der arktische Ozean eisfrei ist und für die wachsende Eismasse genug Nahrung bietet. Bei fortschreitendem Wachstum der neuen arktischen Eisdecke verschlechtern sich die Bedingungen für die Gletscherbildung immer mehr und das Eis schmilzt ab. Damit beginnt das Interglazial.

Gegen diese Theorie ist einzuwenden, daß beide Prozesse, der steuernde und der gesteuerte, von gänzlich verschiedener Zeitdauer sind. Die atmosphärischen Verhältnisse können sich in einigen Monaten ändern. Eine Veränderung der Eisbedeckung des Ozeans dauert Dutzende und die des marinen Wärmehaushaltes hunderte von Jahren. Für den Vorstoß und das Abschmelzen von großen Eismassen müssen aber mehrere zehntausend Jahre angesetzt werden. Würde das Eis zu rasch auf äußere Einflüsse reagieren, so bliebe seine Verbreitung auf enge Grenzen beschränkt.

Auf der Grundlage der Maclaren- und Penck/Hollin-Postulate kann ein anderer Mechanismus von autozyklischen Prozessen zur Erklärung dienen. Aus der Bindung von großen Wassermengen in den Eismassen ergibt sich eine Absenkung des Meeresspiegels um ca. $150 \mathrm{~m}$. Dabei wird ein großer Teil des Barentsmeeres zu Festland. Die verbleibenden Wasserbecken sind so flach, daß sich die Gletscher darüber hinwegschieben können. Diese Bedingungen begünstigen den erneuten Aufbau des Barents-Eisschildes. Daß dieser Eisschild in der letzten Eiszeit und wahrscheinlich auch in den älteren Eiszeiten tatsächlich existierte, wird weitgehend anerkannt (de Geer 1900; Nansen 1904; Blüthgen 1942; Shepard 1963; Grosswald 1967; EMERy 1968). 
Der Skandinavische Eisschild bildete eine hohe Barriere für die atmosphärische W-EZirkulation in den Mittelbreiten. Die atlantischen Luftmassen wurden außerdem von einer stationären Antizyklone über dem Eis an einer Verdriftung nach Osten gehindert. So kam es zu einer Verringerung der Niederschläge in den östlichen und südöstlichen Randgebieten des Eisschildes. Aber infolge der ebenfalls verminderten Verdunstung blieben noch die humiden Klimaverhältnisse, die den weiteren Eiszuwachs begünstigten. Bei einer Annäherung der beiden Eisschilde vom Barentsmeer und von Skandinavien wurde die W-E-Zirkulation noch mehr abgeschwächt und möglicherweise gänzlich gestoppt. Eine EismassenBarriere von Spitzbergen bis zu den Karpathen versperrte den feuchten atlantischen Luftmassen den Weg nach Osten. Die südlich angrenzenden Gebirge waren ebenfalls vergletschert. In dieser Zeit erreichten die Luftemperaturen und die Verdunstung ihre niedrigsten Werte. In den östlich und südlich angrenzenden Gebieten nahmen die Niederschläge ab, und das Klima wurde arid. Damit verringerte sich in den östlichen und südlichen Randgebieten der Eismasse der Eiszuwachs, und schließlich kam es dort zum raschen Zurückschmelzen des Eises, das auch auf die anderen Gletschergebiete der Mittelbreiten in Europa, NW-Sibirien und N-Amerika übergriff. Infolgedessen stieg der Meeresspiegel wieder an, und die Eisbergbildung am Rande des Barents-Eisschildes verstärkte sich, wobei diese Prozesse durch isostatische Senkung des Eis-Untergrundes noch beschleunigt wurden. Dieses führte zum Verschwinden des Barents-Eisschildes. Damit wurde die Interglazialzeit eingeleitet, in der wiederum Verhältnisse herrschten, aus denen sich die nächste Vereisung entwickeln konnte.

Schon NeHriNg (1890) hatte auf Grund von säugetierpaläontologischen Untersuchungen auf die humiden Klimaverhältnisse der Glazialgebiete zu Beginn der Vereisung und auf die Aridität in der Endphase aufmerksam gemacht. M. P. und V. P. GRICHUK (1960) zogen auf der Grundlage von sporen- und pollenanalytischen Untersuchungen eine gleichartige Schlußfolgerung. Die Annahme eines Wechsels von humiden und ariden Klimaverhältnissen während einer Vereisung kann deshalb als Nehring/Grichuk-Postulat bezeichnet werden.

Nach neueren Untersuchungen haben in ganz Nord-Eurasien ähnliche Verhältnisse geherrscht (Frenzel 1968; Gitermann u. a. 1968). Die Lisan-Transgression im Becken des Toten Meeres erfolgte am Beginn der Jungwürm-Eiszeit vor 23-26 000 Jahren, die nachfolgende Regression an deren Ende (NEev \& EMERY 1967). Auch in Nordafrika herrschten am Beginn der Eiszeit relativ humide und am Ende relativ aride Verhältnisse (BUTZER 1958). Die Ergebnisse über die Kaspi-Transgressionen können wegen des zusätzlichen $\mathrm{Zu-}$ flusses von Eisstauwässern einer klimatischen Deutung nicht zugrundegelegt werden (Kvasov 1968).

Der Wechsel von Glazialen und Interglazialen beruht deshalb auf autozyklischen Prozessen. Zur Auslösung ist ein schwacher äußerer Impuls erforderlich. Dieser Impuls war gegeben durch die Abkühlung, die durch periodische Veränderungen der Erdbahnelemente verursacht wurde (Milankovitch-Kurve). Diese Kurve ist für den Zeitabschnitt der letzten 30 Mio. Jahreberechnet worden (Sharaf \& Boudnikova 1969). Die mit der Kurve verbundenen Klimawechsel sind jedoch während großer Zeitabschnitte (auch im Eopleistozän) nicht signifikant. Lediglich im Pleistozän gab es einen Mechanismus, der die extraterrestrisch bedingten Fluktuationen vergrößerte.

\section{Die geologische Zukunft}

Wir haben Grund zu der Annahme, daß die Eiszeit noch nicht zu Ende ist. Die Milankovitch-Kurve ist für den Zeitabschnitt der nächsten Millionen Jahre vorausberechnet worden (Sharaf \& Boudnikova 1969). Danach wird die Abkühlung, die vor ca. 2500 Jahren 
im Subatlantikum einsetzte, noch einige tausend Jahre anhalten. In naher geologischer $\mathrm{Zu}$ kunft wird der Skandinavische Eisschild den kritischen Umfang erreichen, der den o. g. autozyklischen Prozeß einleitet, der dann zur Vereisung führt. Nach mehrmaliger Vereisung wird der Barentssee-Schelf aber so tief ausgeschürft sein, daß der Aufbau der Eisschilde in der gemäßigten Klimazone den Aufbau des Barents-Eisschildes nicht mehr auszulösen vermag. Unter diesen Bedingungen wird der autozyklische Prozeß gestoppt, und es kommt zu einer permanenten Vereisung in der gemäßigten Klimazone.

Daraus kann man den Schluß ziehen, daß drei Haupttypen von Vereisungen vorkommen können: eine permanente Vereisung in den Polargebieten, eine pulsierende und eine permanente Vereisung in der gemäßigten Klimazone. Auf der Südhalbkugel gibt es den erstgenannten Typ nun schon seit einigen Millionen Jahren. Auf der Nordhalbkugel war er jedoch nur für das Eopleistozän charakteristisch. Im Pleistozän gab bzw. gibt es in der gemäßigten Klimazone eine pulsierende Vereisung.

In den nächsten Jahrtausenden kann es zu einer anderen Vereisung kommen. Man muß bedenken, daß sich die menschliche Aktivität gegenwärtig in einer Anreicherung von $\mathrm{CO}_{2}$ in der Atmosphäre und in einer beträchtlichen Energieproduktion auswirkt. Es muß also eine Theorie der allgemeinen atmosphärischen und ozeanischen Zirkulation sowie der Gletscherdynamik entwickelt werden, die nicht nur auf die rezenten Verhältnisse, sondern auch auf die geologische Vergangenheit anwendbar ist. Wenn die Ergebnisse der Berechnungen mit den paläogeographischen Ergebnissen übereinstimmen, kann auf der Grundlage dieser Theorie auch die zukünftige Entwicklung der Verhältnisse in der Atmo- und Hydrosphäre sowie die Gletscherentwicklung berechnet werden, sowohl im Rahmen der natürlichen Weiterentwicklung der Erde als auch im Rahmen des willkürlichen oder planmäßigen Eingreifens des Menschen in die planetarischen Prozesse. Auf der Grundlage dieser Berechnungen könnten ungünstige Veränderungen der Naturvorgänge verhindert und diese schließlich vom Menschen gesteuert werden.

\section{Literatur}

Artem'yev, M. E. \& Artyushrov, Ye. V.: On the origin of the rift depressions. - Proc. USSR Acad. Sci., geol. ser., 4, 58-73, 1968 (in Russisch).

BANDY, O. L.: Foraminiferal definition of the boundaries of the Pleistocene in southern California, USA. - Progr. in Oceanogr., 4, 27-49, 1967.

Baranova, Yu. P. \& BiSke, S. F. et al.: Kaynozoy severo-vostoka SSSR, Moskva 1968.

Bauer, A.: The balance of the Greenland ice sheet. - J. Glaciol., 2, 456-462, 1955.

Blundell, D. J., Griffits, D. H., King, R. F.: Geophysical investigations of buried river valleys around Cardigan Bay. - Geol. J., 6, 161-180, 1969.

BLÜThGEN, J.: Die diluviale Vereisung des Barentsseeschelfes. - Die Naturwissenschaften, 30, 674-679, 1942.

Ввоокs, C. E. P.: Climate through the ages. - London 1950.

ButZer, K. W.: Quaternary stratigraphy and climate in the Near East. - Bonner geogr. Abh., 24, 157 S., 24 Abb., 14 Tab., Bonn 1958.

CEPEK, A. G.: Stand und Probleme der Quartärstratigraphie im Nordteil der DDR. - Ber. deutsch. Ges. geol. Wiss. Reihe A, 12, 375-404, Berlin 1967.

Chizhov, O. P. et al.: Glaciation of Novaya Zemlya. - Moskva 1968 (in Russisch).

Chumakov, I. S.: Pliocene and pleistocene deposits of the Nile valley in Nubia and upper Egypt. Moskva 1967 (in Russisch).

CurRy, R. R.: Glaciation about 3,000,000 years age in the Sierra Nevada. - Science, 154, 770$771,1966$.

Dana, J. D.: Adress of the president of the Association. - Proc. Amer. Assoc. for Adv. of Sci., 9, 21-29, 1855.

De GeER, G.: Om östra Spetsbergens glaciation under istiden. - Geol. Foren. i Stockholm Förhand1. n. 201, 22, 427-436, Stockholm 1900.

EMERY, K. O.: Shallow structure of continental shelves and slopes. - South-eastern Geology, 9, 173-194, 1968. 
Emery, K. O. \& Zarudski, E. F. K.: Seismic reflection profiles along the drill holes on the continental margin of Florida. - Geol. Surv. Profess. Pap., 581-A, Washington 1967.

EwING, J. \& EwING, M.: Sediment distribution on the mid-ocean ridges with respect to spreading of the sea floor. - Science, 156, $\mathrm{n}^{\circ} 3782,1590-1592,1967$.

EwIng, M. \& Donn, W. L.: The theory of ice age. I. - Science, 123, n 3207, 1061-1066, 1956; II. - Science, 127, no $3307,1159-1162,1958$; III. - Science, 152, no 3730, 1706-1712, 1966.

Frenzel, B.: Grundzüge der pleistozänen Vegetationsgeschichte Nord-Eurasiens. - Wiesbaden 1968.

GAKKEL, Ya. Ya. \& DibneR, V. D.: Bottom of the Arctic Ocean. - In: International Dictionary of Geophysics, 1, 152-165, Oxford (Pergamon Press) 1967.

Girdler, R. W., Fairhead, J. D., Searle, R. C. \& Sowerbutts, W. T. C.: Evolution of rifting in Africa. - Nature, 224, $\mathrm{n}^{\circ} 5225,1178-1182,1969$.

Giterman, R. E., Golubeva, L. E., Zaklinskaya, E. D. et al.: The main development stages of the vegetation of North Asia in Anthropogen. - Moskva 1968 (in Russisch).

Glenie, R. C., Schofield, J. C. \& WARD, W. T.: Tertiary sea levels in Australia and New Zealand. - Palaeogeogr., palaeoclim., palaeoecol., 5, 141-163, 1968.

Goodel,H. G., Watkins, N. D., Mather, T. T. \& Koster, S.: The Antarctic glaciation history recorded in sediments of the Southern Ocean. - Palaeogeogr., palaeoclim., palaeoecol., 5, $41-62,1968$.

Gonetsкr, G. I.: O proishozhdenii i vozraste glubokih dolinoobraznyh ponizheniy v relyefe posteli antropogenovyh otlozheniy lednikovoy oblasti. - In: Nizhniy pleistocen lednikovyh rayonov Russkoy ravniny, 17-34, Moskva 1967.

Gregory, J. M.: Raised beaches and variations of sea level. - Scientia (Bologna), 49, 95-104, 1931.

Grichuk, M. P. \& Grichuk, V. P.: Periglacial vegetation on the territory of the USSR. - In: Periglacial phenomena on the territory of the USSR, 66-100, Moskva 1960 (in Russisch).

Grichuk, V. P.: Nizhnyaya graniza chetvertichnogo perioda (sistemy) i eyo stratigraficheskoe polozhenie na Russkoy ravnine. - Trudy Instituta geografii Akademii nauk SSSR, 77, 5-90, 1959.

Gromov, V. I.: Paleontologicheskoe i arheologicheskoe obosnovanie stratigrafii kontinentalnyh otlozheniy chetvertichnogo perioda na territorii SSSR (mlekopitayuschie, paleolit). - Moskva 1948.

Gromov, V. I., Krasnov, I. I., Nikifórova, K. V. \& Shantser, E. V.: Skhema podrazdeleniy antropogena. - Byulleten Komissii po izucheniyu chetvertichnogo perioda, 36, 41-56, 1969.

Grosswald, M. G.: Glaciation of Barents continental shelf in Late Pleistocene and Holocene. Data of Glaciological Studies, Chronicle, Discussions, 13, 52-77, 1967 (in Russisch).

Hamilton, E. L.: Sunken islands of the Mid-Pacific mountains. - Geol. Soc. Amer., Mem., 64, 1956.

Herman, J.: Arctic Paleo-Oceanography in Late Cenozoic time. - Science, 169, 3944, 474-477, 1970.

Hollin, J.: On the glacial history of Antarctica. - J. Glaciol., 4, 173-195, 1962.

HoltedaHL, H.: Some remarks on geomorphology of continental shelves off Norway, Labrador and Southern Alaska. - J. Geol., 66, 461-471, 1958.

Krenke, A. N.: Byudzhet massy i sovremennaya evolucya oledeneniya Zemli Franza-Iosifa, Moskva 1904.

Kvasov, D. D.: Kaspian's balance in Middle Pliocene. - Bull. Moskow Soc. Naturalists, Geol. series, 41, n० 6, 99-114, 1966 (in Russisch).

- : Paleogidrologyiya vostochnoy Evropy $\mathrm{v}$ pozdnechetvertichnoe vremya. - In: Doklady na ezhegodnyh chteniyah pamyati L. S. Berga, VIII-XIV, 65-80, Leningrad 1968.

- : Limnological hypothesis of the origin of hot brines in the Red Sea. - Nature, 221, $\mathrm{n}^{\circ} 5183$, 850-851, 1969.

Kvasov, D. D., Ananova, E. N., Borisov, A. A. \& Dibner, V. D.: On palaeogeography Eastern Europe in the akchagilsko-apsheronsky period. - Vestnik Leningradskogo universiteta, $\mathrm{n}^{\circ} 6$, 142-151, 1969 (in Russisch).

Maclaren, C.: The glacial theory of professor Agassiz. - Amer. J. Sci. and Arts, 42, 346-365, 1842.

Malachovsky, D. B., Markov, K. K. (editors): Geomorphologie et les dépóts quarternaires du nord-ouest de la partie européenne de l'URSS. - Leningrad 1969 (in Russisch).

Markov, K. K., Lazukov, G. I. et al.: The Quaternary period, v. I. Moskva 1965 (in Russisch).

Menard, H. W.: Marine geology of the Pacific. - New York 1964.

Mercer, J. H.: Glaciation of southern Argentina more than two million years ago. - Science, 164, n ${ }^{\circ} 3881,823-825,1969$. 
Nansen, F.: The bathymetrical features of the North Polar seas, with a discussion of the continental shelves and previous oscillations of shore-line. - The Norwegian North Polar Expedition 1893-1896. - Scienific results, 4, $\mathrm{n}^{\circ} 13$, Christiania 1904.

Neev, D., Emery, K. O.: The Dead Sea. - Bull. Geol. Surv. Israel, 41, Jerusalem 1967.

Nehring, A.: Über Tundren und Steppen der Jetzt- und Vorzeit mit besonderer Berücksichtigung ihrer Fauna. - Berlin (Dümmler) 1890.

Nikonov, A. A.: On the old valleys of the north-eastern part of Baltic Shield. - Doklady A. N. SSSR, 177, $\mathrm{n}^{\circ}$ 5, 1155-1158, 1967 (in Russisch).

Penck, A.: Die Ursachen der Eiszeit. - S.B. preuß. Akad. Wiss., phys.-math. K1., n 6, 76-85, Berlin 1928.

Petrov, O. M.: Anthropogen marine mollusks of Northern Eurasia and their importance for stratigraphy. - In: The main problems of Anthropogen geology of Eurasia, 94-100, Moskva 1969 (in Russisch).

RüHLE, E.: Relief of Pre-Quaternary rocks and its effect in the development and character of glacial deposits. - In: Last European Glaciation, 66-69, Moskva 1965 (in Russisch).

Sharaf, S. G. \& Boudnikova, N. A.: Secular perturbations in the elements of the Earth's orbit and astronomical theory of climate variations. - Trudy Instituta teoreticheskoy astronomii, 14, 48-104, 1969 (in Russisch).

ShePARD, F. P.: Submarine geology. - New York 1963.

SHIK, S. M.: Dolednikovaya gidrograficheskaya set yugo-zapadnogo Podmoskovya i eyo razvitie v pleistozene. - Materialy Vsesoyuznogo soveschaniya po izucheniyu chetvertichnogo perioda, 2, 259-263, Moskva 1961.

Steuerwald, B. A., Clark, D. L. \& Andrew, J. A.: Magnetic stratigraphy and faunal patterns in Arctic Ocean sediments. - Earth and Planet. Sci. Lett., 5, 2, 79-85, 1968.

TANnER, W. F.: Multiple influences on sealevel changes in the Tertiary. - Palaeogeogr., palaeoclim,. palaeoecol., 5, 165-171, 1968.

Trorzky, S. L.: Chetvertichnye otlozheniya i relyef ravninnyh poberezhiy Eniseyskogo zaliva i prilegayuschey chasti gor Byrranga. - Moskva 1966.

Udrnzev, G. B.: Bottom relief of the Okhotsk Sea. - Trudy Instituta okeanologii A. N. SSSR, 22, 3-76, 1957 (in Russisch).

Uflyand, A. K. et al.: Depressions of Baikalian type in Northern Mongolia. - Bull. Moskow Soc. Naturalists, Geol. series, 44, n 6, 5-22, 1969 (in Russisch).

Valentin, H.: Der Nordseeraum an der Wende Tertiär-Quartär. - Die Erde, 3-4, 285-303, Berlin 1951-1952.

VASILYev, Yu. M.: Formation des dépôts anthropogénes des zones glaciaires et extraglaciaires. Moskva 1969 (in Russisch).

Volkov, I. A. et al.: Loess-like cover deposits and paleogeography of the south-west of West Sibiria in Pliocene-Quaternary time. - Novosibirsk 1969 (in Russisch).

Voorthuysen, J. H. van: Crustal movements of the North Sea basin during Pliocene and early Pleistocene time. - Geol. en Mijnb. N. S., 16, 165-172, 1954.

WENsink, H.: Paleomagnetic stratigraphy of younger basalts and intercalated plio-pleistocene tillits in Iceland. - Geol. Rdsch., 54, 364-384, Stuttgart 1965.

Manuskr. eingeg. 13. 12. 1970.

Anschrift des Verf.: D. D. Kvasov, Laboratory of Limnology, Academy of Science, USSR, Leningrad P-46, Petrovskaja ul. 3a. 